\title{
Narrowband HST Images of Microstructures in Planetary Nebulae
}

\section{B. Balick ${ }^{1}$, J. Alexander ${ }^{1}$, A. Hajian ${ }^{2}$, Y. Terzian ${ }^{3}$, M. Perinotto ${ }^{4}$ and P. Patriarchi ${ }^{5}$}

${ }^{1}$ University of Washington; ${ }^{2}$ U.S. Naval Observatory; ${ }^{3}$ Cornell University;

${ }^{4}$ Universita degli Studi di Firenze; ${ }^{5}$ GNA/CGN, Osservatorio Astrofisico d'Arcetri

Hubble Space Telescope narrowband images of four PNe resolve the microstructures in four PNe known to contain FLIERs (e.g. Balick et al 1994). FLIERs are seen to fall into two morphological groups: very low ionization clumps of small knots with cometary-like tails, and sinewy transverse filaments with fainter extensions pointing outward from the nucleus. Generally speaking, FLIERs exhibit [NII] $\lambda 6584 / \mathrm{H} \alpha>1$. They are marginally detectable in $\mathrm{H} \alpha$ above the bright background of nebular emission, and all but invisible in [O III] $\lambda 5007 \mathrm{~A}$. In every case the star-facing side of the structure is on the order of a pixel in width (about $10^{15} \mathrm{~cm}$ ). A decreasing ionization gradient is seen [O I] $66300 \mathrm{~A},[\mathrm{~S}$ II] $\lambda 6717+31 \mathrm{~A}$, and [N II] $\lambda 6584 \mathrm{~A}$ images.

The outward pointing tails and extensions, the decreasing radial ionization, and the absence of a leading bow shock all argue that the previous paradigm for FLIERs as Nrich stellar "spitballs" is inapplicable. Instead, it appears that the FLIERs are associated with dense neutral knots which are photoionized by and immersed in an outward flowing invisible wind from the central star.

The morphological and kinematic data for FLIERs suggest that they are mass-loaded flows (e.g. Arthur et al 1994) in which neutral ablated gas is accelerated by and incompletely mixed with the ablating wind. Such a model is perhaps in accord with the cometary nebulae observed in Abell 30 (Borkowski et al 1995). Although cometary nebulae are known to exist in NGC 7293 (O'Dell and Hadron 1996) and FG 1 (Palmer et al. 1996) - suggesting that embedded knots are not unusual in $\mathrm{PNe}$ - the high velocities of the emitting gas associated with FLIERs that they are immersed in a high speed wind, perhaps like the wind in Abell 30.

In any case, the formation of these knots remains an enigma whose resolution might be found in the development of structure during the cool protoPN phase of evolution (Frank et al., this conference). A way of explaining the apparently high N/O ratio in FLIERs is still being sought (Riera et al. this conference).

Color figures of the WFPC2 images are available on the WWW at http: / /www.astro.washington. edu/balick/W_F_P_C_2/N_3_2_4_2/N_3_2_4_2_3color.gif http: / /www . astro.washington. edu/balick/WF_P_C_2/N_6_8_2_6/N_6_8_2_6_3color.gif http: / / www . astro. washington. edu/balick/W_F_P_C_2/N_7_0_0_9/W_F_7_0_0_9_3color.gif http: / / www .astro. washington. edu/balick/W_F_P_C_2/N_7_6_6_2/N_7_6_6_2_3color.gif

\section{REFERENCES}

Arthur, S.J., Dyson, J.E., \& Hartquist, T.W. 1994, MNRAS, 269, 1117.

Balick, B., Perinotto, M., Maccioni, A., Terzian, Y., \& Hajian, A., 1994, ApJ, 424, 800.

Borkowski, K.J., Harrington, J.P., \& Tsvetanov, Z.I., 1995, ApJ, 449, L143.

O'Dell, C.R. \& Hadron, K.D., 1996, AJ, 111, 2320.

Palmer, J.W., Lopez, J.A., Meaburn, J., \& Lloyd, H.M., 1996, A\&A, 307, 225. 